\title{
Geo-effectiveness and radial dependence of magnetic cloud erosion by magnetic reconnection
}

\author{
Benoit Lavraud, ${ }^{1,2}$ Alexis Ruffenach, ${ }^{1,2}$ Alexis P. Rouillard, ${ }^{1,2}$ Primoz Kajdic, ${ }^{1,2}$ \\ Ward B. Manchester, ${ }^{3}$ and Noé Lugaz ${ }^{4}$ \\ Received 25 June 2013; revised 23 October 2013; accepted 10 December 2013; published 15 January 2014.
}

[1] Magnetic flux erosion by magnetic reconnection occurs at the front of at least some magnetic clouds (MCs). We first investigate how erosion influences the geo-effectiveness of MCs in a general sense and using a south-north magnetic polarity MC observed on 18-20 October 1995. Although the magnetic shear at its front may not be known during propagation, measurements at $1 \mathrm{AU}$ show signatures of local reconnection. Using a standard MC model, an empirical model of the geomagnetic response (Dst), and an observational estimate of the magnetic flux erosion, we find that the strength of the observed ensuing storm was $\sim 30 \%$ lower than if no erosion had occurred. We then discuss the interplay between adiabatic compression and magnetic erosion at the front of MCs. We conclude that the most geo-effective configuration for a south-north polarity MC is to be preceded by a solar wind with southward IMF. This stems not only from the formation of a geo-effective sheath ahead of it but also from the adiabatic compression and reduced (or lack thereof) magnetic erosion which constructively conspire for the structure to be more geo-effective. Finally, assuming simple semiempirical and theoretical Alfvén speed profiles expected from expansion to $1 \mathrm{AU}$, we provide first-order estimates of the erosion process radial evolution. We find that the expected reconnection rates during propagation allow for significant erosion, on the order of those reported. Calculations also suggest that most of the erosion should occur in the inner heliosphere, and up to $\sim 50 \%$ may yet occur beyond Mercury's orbit.

Citation: Lavraud, B., A. Ruffenach, A. P. Rouillard, P. Kajdic, W. B. Manchester, and N. Lugaz (2014), Geo-effectiveness and radial dependence of magnetic cloud erosion by magnetic reconnection, J. Geophys. Res. Space Physics, 119, 26-35, doi:10.1002/2013JA019154.

\section{Introduction}

[2] Coronal mass ejections (CMEs) are central to solar wind-magnetosphere interaction [Zhang and Burlaga, 1988; Gosling, 1993; Farrugia et al., 1993; Lavraud and Borovsky, 2008]. They are the drivers of the strongest geomagnetic storms and associated hazards in the near-Earth environment. Their geo-effectiveness is very hard to predict. This is not only due to the complexity of the processes that

\footnotetext{
${ }^{1}$ Institut de Recherche en Astrophysique et Planétologie, Université de Toulouse, Toulouse, France.

${ }^{2}$ Centre National de la Recherche Scientifique, UMR 5277, Toulouse, France.

${ }^{3}$ Department of Atmospheric, Oceanic, and Space Sciences, University of Michigan, Ann Arbor, Michigan, USA.

${ }^{4}$ Space Science Center, University of New Hampshire, Durham, New Hampshire, USA.

Corresponding author: B. Lavraud, Institut de Recherche en Astrophysique et Planétologie, Université de Toulouse, 9 Avenue du Colonel Roche, BP 44346, FR-31028 Toulouse CEDEX 4, France. (Benoit.Lavraud@irap.omp.eu)

(C)2013. American Geophysical Union. All Rights Reserved. 2169-9380/14/10.1002/2013JA019154
}

occur at the Sun during their emergence (convection, reconnection, heating, acceleration/deceleration, deflection, rotation, and else) but is also largely the result of various processes that occur during their propagation to the Earth. The formation of shocks and sheath [Gosling et al., 1990], the orientation and potential crossing of the CME legs [e.g., Möstl et al., 2010; Owens et al., 2012], as well as compression effects at their back owing to trailing high-speed winds [Fenrich and Luhmann, 1998; Rouillard et al., 2010] have been much studied and have strong implications for CME geo-effectiveness. Here we highlight the geo-effective impact - which has not been addressed so far - of the occurrence of magnetic reconnection at the front of CMEs which erodes away part of the magnetic flux impinging on the Earth's magnetosphere.

[3] Magnetic reconnection is ubiquitous in the solar wind [Farrugia et al., 2001; Gosling et al., 2005, 2006; Phan et al., 2006; Davis et al., 2006; Huttunen et al., 2008; Eriksson et al., 2009; Lavraud et al., 2009]. Among the key signatures to be observed in situ is the presence of a velocity jet bounded by bifurcated current sheets satisfying the Walén relation. It has been demonstrated to occur at various key boundaries, including the heliospheric current 
(a)

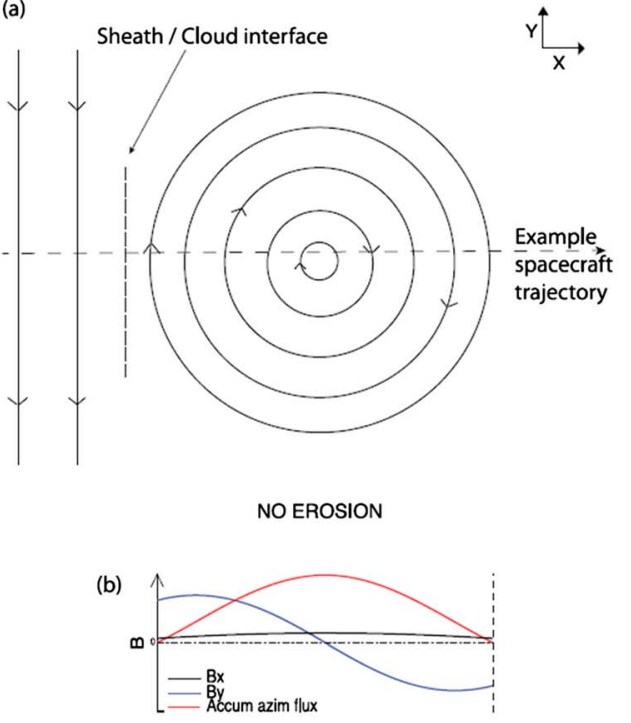

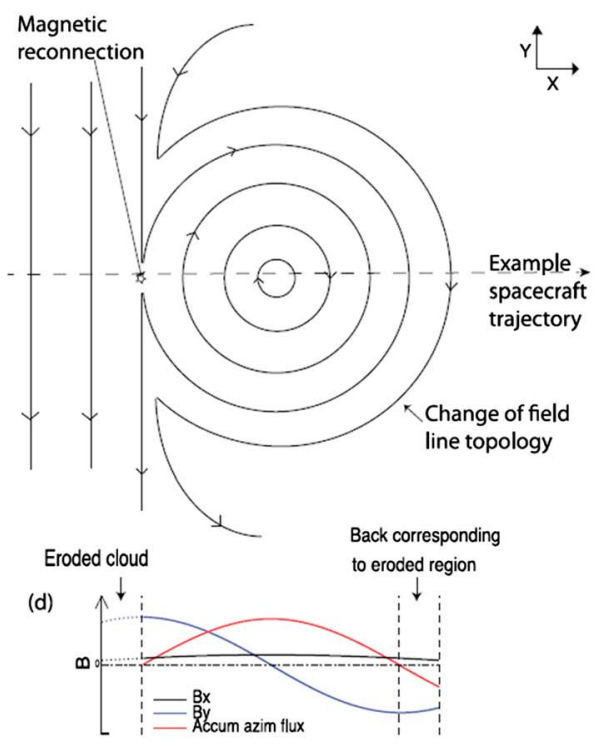

Figure 1. Schematic representing the magnetic structure of (a) noneroded and (c) eroded MCs together with (b and d) the expected variations in the magnetic field components and accumulated azimuthal magnetic flux. The analysis needs to be made in the proper MC coordinate system as implicit here. Figure from Ruffenach et al. [2012].

sheet [Gosling et al., 2006; Lavraud et al., 2009] and within [Gosling and Szabo, 2008] and at the front boundary of MCs [Ruffenach et al., 2012]. Reconnection is particularly frequent in low $\beta$ plasma (e.g., MCs), even for low magnetic shear [Gosling and Szabo, 2008], which fact may be explained by the dependence of magnetic reconnection on plasma $\beta$ and magnetic shear [Swisdak et al., 2003, 2010].

[4] If it occurs at the front boundary of a magnetic cloud (MC), then magnetic reconnection erodes away part of the magnetic flux contained in the MC [McComas et al., 1988; Dasso et al., 2006, 2007; Möstl et al., 2008; Ruffenach et al., 2012]. This erosion process is schematically illustrated in Figure 1, taken from Ruffenach et al. [2012]. Most of these previous studies have investigated the influence of such magnetic erosion process on the total and azimuthal magnetic flux contents of MCs which had a north-south (NS) magnetic field polarity (e.g., leading northward and trailing southward interplanetary magnetic field (IMF) in GSE (geocentric solar ecliptic) coordinates). Erosion has been also studied using global magnetohydrodynamic (MHD) simulations [Schmidt and Cargill, 2003; Taubenschuss et al., 2010], pointing in particular to the fact that the efficiency of the reconnection process increases with the relative speed of the MC with respect to the ambient solar wind.

[5] In Ruffenach et al. [2012], given the transit time of the MC from the Sun to the Earth and the estimate of the eroded magnetic flux, the average reconnection rate required to account for the estimated erosion during propagation was found to be in the range of $0.12-0.22 \mathrm{mV} / \mathrm{m}$, and it was $0.45-0.51 \mathrm{mV} / \mathrm{m}$ for the events studied in Dasso et al. [2006, 2007]. They noted that on average, these estimations are significantly larger than those given in case studies of reconnection exhausts observed in situ at L1 by Davis et al. [2006], Phan et al. [2006], and Wang et al. [2010] (0.02, 0.03 , and $0.05-0.08 \mathrm{mV} / \mathrm{m}$, respectively). Despite this apparent inconsistency, we will argue in section 5 that reconnection rates during propagation from the Sun to $1 \mathrm{AU}$ may be sufficient for erosions on the order of those estimated here and in previous work [cf. Ruffenach et al., 2012].

[6] In the present paper we focus on the analysis of the influence of such magnetic erosion on the amount and duration of southward-directed magnetic flux (and driver electric field) and subsequent geo-effectiveness as given by both measured and modeled ring current intensities through the Dst index [e.g., Burton et al., 1975; O'Brien and McPherron, 2000]. This is done in a generic fashion in section 2 and then using a case study in section 3. Section 4 specifically discusses the interplay expected between erosion and compression, while section 5 presents estimates of the radial evolution of the erosion process. Section 6 provides some discussion in view of the limitations of the approach used, and section 7 concludes this paper.

\section{Method and Illustration of the Influence of MC Erosion on the Dst Index}

[7] Estimation of the influence of MC erosion on ensuing geomagnetic storm strength is performed here using a simple, parametric approach based on an MC analytical model and a semiempirical model of geomagnetic response. We use a Lundquist flux rope model [Lundquist, 1950], with a baseline of $24 \mathrm{~h}$, maximum magnetic field $B_{\mathrm{MAX}}=30 \mathrm{nT}$, and constant bulk speed $|V|=400 \mathrm{~km} / \mathrm{s}$. We take the main $\mathrm{MC}$ axis along the $Y_{\mathrm{GSE}}$ direction, so that the $\mathrm{MC}$ has a southnorth magnetic polarity with its geo-effective part being the front first half of the MC. These properties were chosen because they give an electric field $E_{y}$ (or $V B_{Z}$ parameter) on the order of $\sim 9 \mathrm{mV} / \mathrm{m}$ in the front part of the $\mathrm{MC}$, similar to that observed in the case studied in section 3 .

[8] We then make use of the semiempirical model of the Dst index as parameterized by O'Brien and McPherron [2000], based on the early work by Burton et al. [1975]. 


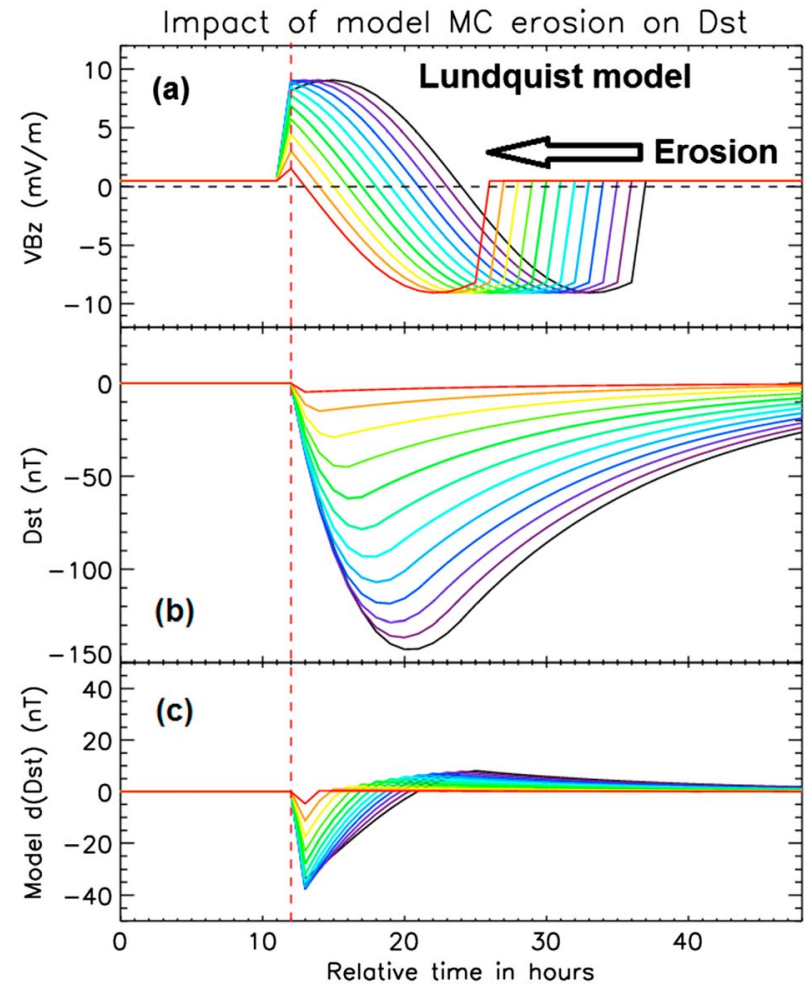

Figure 2. Parametric study of expected $\mathrm{MC}$ erosion impact on ensuing storm strength. (a) $V B_{Z}$ parameter (i.e., solar wind electric field) based on a Lundquist flux rope model with $B_{\mathrm{MAX}}=23 \mathrm{nT}$ and $|V|=400 \mathrm{~km} / \mathrm{s}$. (b) Expected Dst index using the semiempirical model of $O$ 'Brien and McPherron [2000] using a ram pressure $P_{\mathrm{RAM}}=1 \mathrm{nPa}$. (c) Rate of the Dst change from the semiempirical model. The black lines show the results for a noneroded flux rope. Various levels of $\mathrm{MC}$ erosion are shown with colored curves (from purple to red), based on $1 \mathrm{~h}$ increments.

The Dst index quantifies the average variation of the horizontal magnetic field component measured on ground near the equator, as induced by enhancement in the ring current encircling the Earth during storms. The contribution from the magnetopause current system needs to be removed prior to analyzing $D s t$ as an actual index representing the strength of inner magnetospheric currents. This contribution is given as a function of solar wind dynamic pressure, as follows:

$$
D s t^{*}=D s t-7.26 \sqrt{P_{\text {ram }}}+11
$$

[9] This corrected index is called $D s t^{*}$. The underlying analytical formula in the semiempirical model [equation (2)] is made of an injection term (first right-hand term) and a decay term (second right-hand term) as follows:

$$
\frac{d}{d t} D s t^{*}(t)=Q(t)-\frac{D s t^{*}(t)}{\tau}
$$

where $\tau$ is a decay time scale in hours. Although the decay time scale has been shown to depend on the Dst value [Dasso et al., 2002; Aguado et al., 2010], we here use a constant decay time scale as parameterized by $O$ 'Brien and McPherron [2000] since our analysis does not focus on storm recovery phase. Magnetospheric and ring current driving are intimately related to the electric field $\left(V B_{Z}\right.$ in $\left.\mathrm{mV} / \mathrm{m}\right)$ and dynamic pressure $\left(P_{\text {ram }}\right.$ in $\left.\mathrm{nPa}\right)$ of the impinging solar wind. The injection term $Q\left(\mathrm{nT} \mathrm{h}^{-1}\right)$ is built as

$$
\begin{array}{ll}
Q=0 & V B z \leq 0.49 \mathrm{mV} / \mathrm{m} \\
Q=-4.4(V B z-0.49) & V B z>0.49 \mathrm{mV} / \mathrm{m}
\end{array}
$$

The decay time scale $\tau$ (in hours) from equation (2) has the following parameterization:

$$
\begin{aligned}
\tau & =2.40 e^{6.66 / 4.69} & & B z \geq 0 \mathrm{nT} \\
\tau & =0.40 e^{9.74 /(4.69+V B z)} & & B z<0 \mathrm{nT}
\end{aligned}
$$

The $D s t^{*}$ index is calculated at time $t+\Delta t(\Delta t$ is $1 \mathrm{~h})$ through direct integration of equation (2) using the time series of $V B_{Z}$. Because our analysis is primarily qualitative, we will present only $D s t^{*}$ values using a small $P_{\text {ram }}=1 \mathrm{nPa}$, consistent with the small value observed within the MC of 18-20 October 1995.

[10] Figure 2 illustrates the impact of the erosion process on the $D s t^{*}$ index for the given MC model. Figure 2a shows the $V B_{Z}$ parameter, first positive then negative, as expected for a south-north polarity MC. The black line shows the noneroded $\mathrm{MC}$, and every subsequent colored curve corresponds to an eroded $\mathrm{MC}$ in increments of $1 \mathrm{~h}$. The corresponding $D s t^{*}$ response according to the O'Brien and McPherron [2000] model is given in Figure 2b, and its rate of change is given in Figure 2c. This figure clearly highlights the direct impact of the erosion process on the resulting storm strength. Although the Dst model used is not linear by construction (owing to the decay term), it is a direct and monotonic function of the $V B_{Z}$ amplitude and duration. Although overall trivial, this plot gives a direct and clear feeling about the potential importance of the erosion process. Using multispacecraft observations of the 19-20 November 2007 MC, Ruffenach et al. [2012] showed an erosion of almost $50 \%$ of its azimuthal magnetic flux at the front (corresponding to $\sim 25 \%$ of the unsigned magnetic flux measured over the full MC sample). This particular MC had a northsouth orientation, and the erosion thus had little impact at the Earth. Had it been of reversed polarity, Figure 2 shows that the ensuing storm would have been significantly reduced owing to erosion.

\section{The South-North Polarity MC of $\mathbf{1 8}$ October 1995}

[11] We now illustrate the erosion process using an actual south-north polarity MC which occurred on 18-20 October 1995 and drove a storm with minimum $D s t^{*}$ of $\sim 123 \mathrm{nT}$. Figure 3 shows the solar wind data for this event. The event is labeled "MC," and its boundaries are given with dashed vertical lines. We define them at 19:01 UT on 18 October and 01:37 UT on 20 October, similar to Larson et al. [1997]. The leading shock and sheath are marked. The MC core magnetic field strength is on the order of 20-25 nT. The speed is $\sim 400 \mathrm{~km} / \mathrm{s}$ and constant within the MC. The $V B_{Z}$ parameter is shown in Figure $3 \mathrm{~b}$ and, on purpose, is similar to that used in Figure 2. Despite a small temporal 


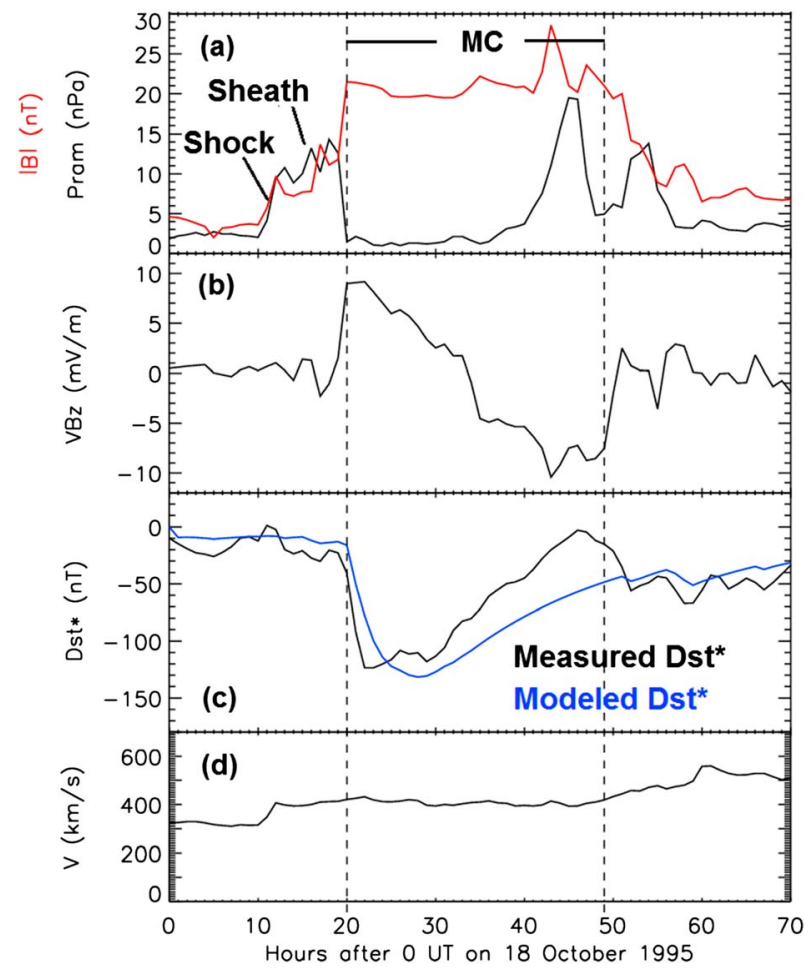

Figure 3. OMNI solar wind data from the 18-20 October $1995 \mathrm{MC}$ and storm. (a) Ram pressure and magnetic field magnitude (red). (b) $V B_{Z}$ parameter, i.e., the solar wind electric field. (c) Measured (black) and modeled (blue) $D s t^{*}$ indexes, i.e., the Dst index corrected for the contribution from the magnetopause current contribution (cf. text for details). (d) Solar wind proton speed. The CME (magnetic cloud) and its leading shock and sheath are highlighted.

lag, the modeled $D s t^{*}$ following $\mathrm{O}^{\prime}$ Brien and McPherron [2000] is reproducing well the observed value and, in particular, its magnitude.

[12] This event was chosen because it has a clean magnetic cloud structure, rather clear boundaries, and a main axis orientation as determined from minimum variance analysis (MVA) close to $Y_{\mathrm{GSE}}$. The axis orientation we use is $(0.37$, $-0.91,-0.19)$, or $\theta=-18^{\circ}$ and $\varphi=292^{\circ}$, in GSE coordinates. This MC has been studied by several authors [Lepping et al., 1997; Larson et al., 1997; Janoo et al., 1998; Collier et al., 2001; Hidalgo et al., 2002; Dasso et al., 2006]. The timing of the end boundary of the MC and the main axis orientation differ slightly between these studies (cf. details in Dasso et al. [2006]). However, the axis orientations found are all well oriented toward $Y_{\mathrm{GSE}}$, and, as can be seen in Dasso et al. [2006, Figure 5], even using an earlier MC end boundary yields a significant asymmetry in azimuthal magnetic field structure, compatible with erosion as also reported next in our analysis.

[13] Figure 4 shows the higher-resolution magnetic field data in the proper MC frame of reference. This frame is defined [e.g., Dasso et al., 2006; Ruffenach et al., 2012] so that $Z_{\text {cloud }}$ is the main axis of the MC. The direction $d$ is defined by the rectilinear trajectory of the spacecraft $\left(X_{\mathrm{gse}}\right), Y_{\text {cloud }}$ is in the direction $Z_{\text {cloud }} \times d$, and $X_{\text {cloud }}$ completes the righthanded coordinate system (cf. illustration in Figure 1). The
MC main axis is found using a combined "nested-bootstrap" minimum variance analysis (MVA) on the normalized magnetic field inside the MC [cf. Ruffenach et al., 2012]. We apply a bootstrap method [e.g., Kawano and Higuchi, 1995] with 1000 random data resamplings. We repeat this for seven nested time intervals within the MC separated by $10 \mathrm{~min}$ : each of the seven time intervals begins $10 \mathrm{~min}$ after the previous and ends $10 \mathrm{~min}$ before. This resampling is meant to assess the magnetic field intrinsic variability impact on the main $\mathrm{MC}$ axis determination and, in turn, the impact on the asymmetry in azimuthal magnetic flux.

[14] The black line in Figure 4 shows the azimuthal magnetic field component in the average frame of reference (from all MVA analyses). Using all axes determined by the aforementioned nested-bootstrap MVA method, the colored curves display the accumulated magnetic flux per unit length $\left(F_{y} / L_{\text {in }}\right)$ following the direct method from Dasso et al. [2007], defined as follows:

$$
\left(F_{y(x)} / L_{\text {in }}\right)=\int_{\text {in }}^{t(x)} B_{y, \text { cloud }}\left(t^{\prime}\right) V_{x, \text { cloud }}\left(t^{\prime}\right) \mathrm{d} t^{\prime}
$$

where $t_{\text {in }}$ is the time of the MC's front boundary, and $B_{y}$ and $V_{x}$ are the respective components of the magnetic field and velocity in the MC frame (cf. above).

[15] The color curves in Figure 4 cross the $X$ axis, on average, $\sim 4 \mathrm{~h}$ before the end of the magnetic cloud. This asymmetry in accumulated magnetic flux, as discussed in the introduction and illustrated in Figure 1, is taken as the signature of magnetic flux erosion by magnetic reconnection at the front of this MC. Based on this analysis and following the methods detailed in Dasso et al. [2006], estimates of the eroded azimuthal magnetic flux per unit length is $\sim 1.28 \times 10^{21} \mathrm{Mx} / \mathrm{AU}(24.0 \%$ of the total azimuthal flux), and the eroded total axial magnetic flux is $\sim 0.46 \times 10^{21} \mathrm{Mx}(24.1 \%$ of the total axial flux). It should be

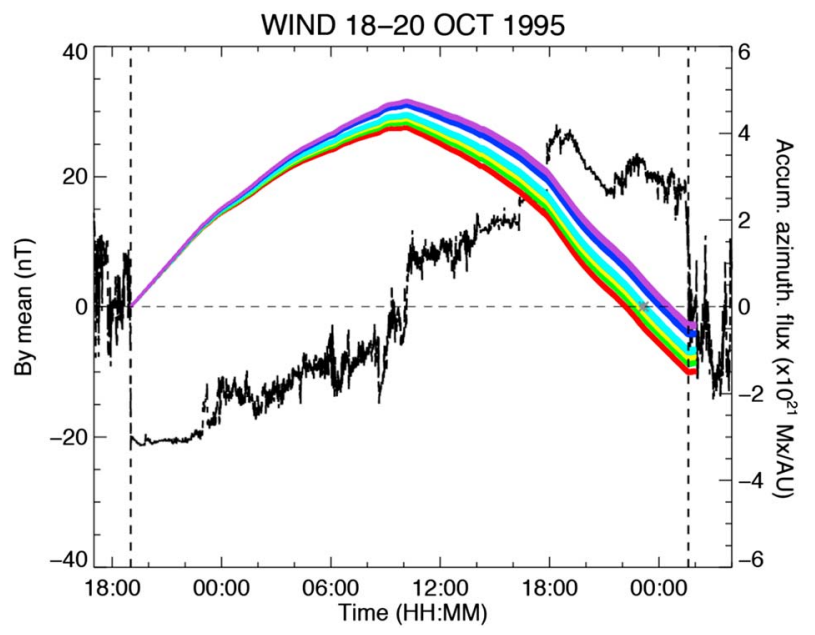

Figure 4. High-resolution azimuthal magnetic field component (black line, left axis) in the average (from MVA analysis; cf. text for details) MC coordinate system for the 18-20 October 1995 event. The colored curves show the integrated azimuthal magnetic flux using the various MC axis orientations resulting from applying a nested-bootstrap MVA method. The observed asymmetry with a $\sim 4 \mathrm{~h}$ long back region of azimuthal flux excess is deemed to correspond to erosion at the front of the MC (cf. also Figure 1). 


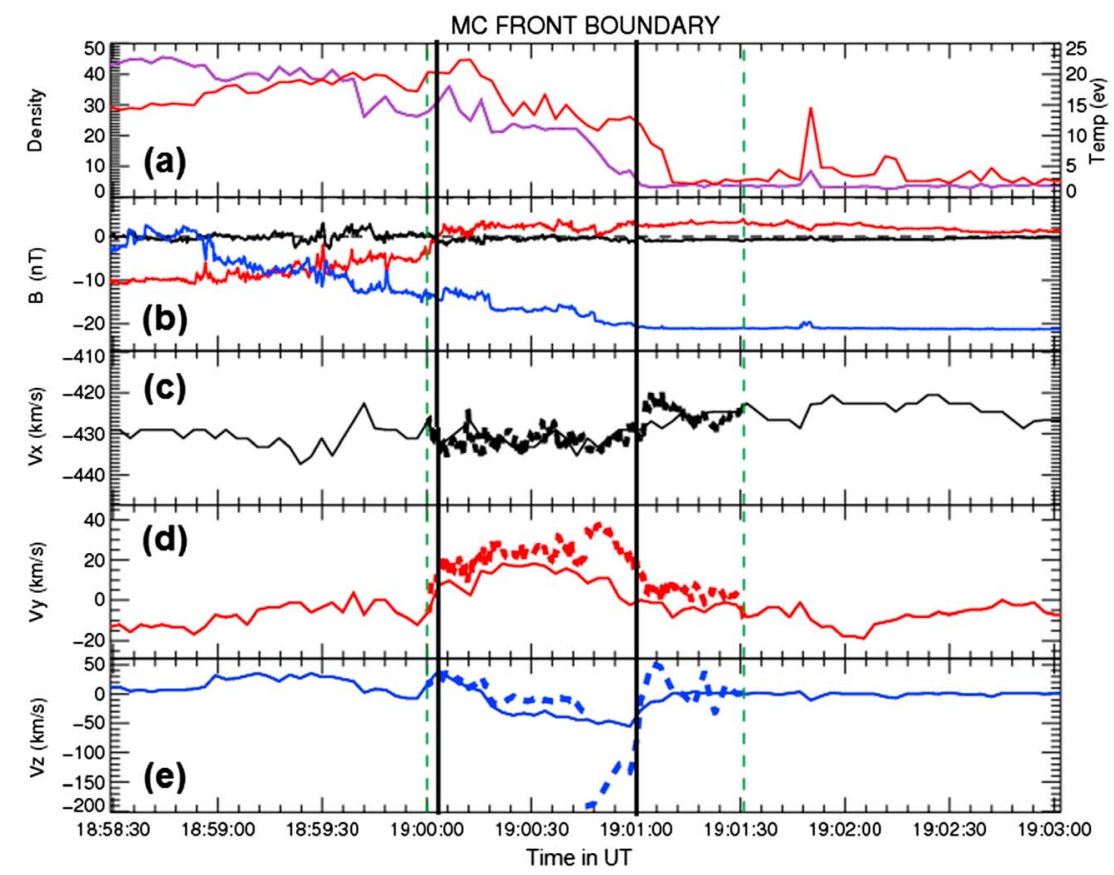

Figure 5. High-resolution magnetic field and velocity data for a short interval at the front boundary of the 18-20 October $1995 \mathrm{MC}$ : (a) (left) ion density and (right) temperature, (b) magnetic field components ( $X$ : black, $Y$ : red, $Z$ :blue), and (c, d, and f) the $X, Y$, and $Z$ velocity components. The GSE system is used here. A possible reconnection exhaust, which shows both bifurcated current and a velocity jet within it, is highlighted with the black vertical thick lines. A Walén test was performed inward from the two green vertical dashed lines each side of the exhaust with successively correlated and anticorrelated changes in the $V-B$ Walén relationship. The expected velocity changes (from the magnetic field changes) in each component are shown with the dashed curves on top of the measured velocity component.

noted that for the same MC, Dasso et al. [2006] found even more erosion than that reported here using an earlier $\mathrm{MC}$ rear boundary crossing time.

[16] Figures $5 \mathrm{a}-5 \mathrm{e}$ show a zoom in of $3 \mathrm{~s}$ resolution proton density, temperature, velocity, and magnetic field data from the Wind spacecraft at the front boundary of the $\mathrm{MC}$, in the GSE coordinate system. The boundary shows a first clear rotation of the magnetic field $B_{Y}$ component at the first thick vertical black line. At that time, the spacecraft enters a transition region with both mixed properties in density and temperature (i.e., intermediate values in Figure 5a) and a clear velocity jet of $\sim 20 \mathrm{~km} / \mathrm{s}$ and $\sim 50 \mathrm{~km} / \mathrm{s}$ in the $V_{Y}$ and $V_{Z}$ components, respectively. A second, fainter magnetic boundary is seen at the second thick vertical line, which corresponds to the end of the plasma mixing region and of the velocity jet. Applying the Walén test inward from the dashed green lines (i.e., at times outside the exhaust), with first correlated and then anticorrelated changes between the velocity and magnetic field components (cf. Phan et al. [2006] for details on such Walén test application), we observe a good correlation between the measured and predicted velocity changes for the first current sheet (thick dashed curves in Figures 5c-5e). Despite the direction being correct, the predicted velocity changes at the second current sheet are not as good: the changes are expected to be larger than the actual observations. We note that this particular boundary is highly asymmetric, with significantly different densities (from $50 \mathrm{~cm}^{-3}$ down to $2 \mathrm{~cm}^{-3}$ over the interval shown) and magnetic field magnitudes each side. The Alfvén speed is thus extremely high on the MC side (right-hand side). We speculate that this strong asymmetry is related to the inaccuracy of the Walén test, but this topic is left for future studies. In summary, despite the partial failure of the Walén test on one side, its success on the other side together with the presence of two current sheets bounding a significant velocity jet with mixed plasma properties argues in favor of ongoing magnetic reconnection at this boundary.

\section{Interplay Between Magnetic Erosion and Adiabatic Compression}

[17] We now investigate what the geomagnetic response would have been had the MC of 18-20 October 1995 not been eroded. Figure 6 shows modeling results akin to those performed in Figure 2. The black lines in Figure 6 show results for a Lundquist flux rope model with $B_{\mathrm{MAX}}=23 \mathrm{nT}$, solar wind speed $|V|=400 \mathrm{~km} / \mathrm{s}$, and ram pressure $P_{\text {ram }}=1$ $\mathrm{nPa}$. The MC duration is set to $30 \mathrm{~h}$. The choice of these properties will be made evident in the following.

[18] The magnetic field magnitude in the 18-20 October $1995 \mathrm{MC}$ does not show a maximum value near the center (cf. Figure 3a), as would be expected from a pure Lundquist solution. This most likely owes to compression of the MC. This is common, particularly at the fronts of MCs which expand into a slower solar wind, and is the case here with the formation of a shock and sheath ahead. So as to best reproduce the characteristics of this particular MC for modeling purposes, the blue lines in Figure 6 correspond to a "compressed" Lundquist model. This is done assuming a constant magnetic field strength throughout the MC, as 
Impact of MC compression and erosion on Dst



Figure 6. Estimates of the effect of compression and erosion on ensuing storm strength, based on input parameters consistent with the 18-20 October $1995 \mathrm{MC}$. A solar wind speed of $400 \mathrm{~km} / \mathrm{s}$ and a ram pressure of $1 \mathrm{nPa}$ are used. The black curves are based on a pure Lundquist flux rope model with $B_{\mathrm{MAX}}=23 \mathrm{nT}$ and a $30 \mathrm{~h}$ duration. The blue lines correspond to a "compressed" Lundquist model, and the red line corresponds to an eroded (by $4 \mathrm{~h}$ ) compressed model. (a) Magnetic field magnitude. (b) $V B_{Z}$ parameter, i.e., the solar wind electric field. (c) Modeled Dst index and (d) its rate of change (cf. text for details).

observed in our event, and by reducing the duration of the MC (black lines) so that the total magnetic flux remains equal to the noncompressed Lundquist solution (cf. Démoulin and Dasso [2009] for more detailed modeling of MC compression during propagation). The red lines in Figure 6 show the results now assuming a compressed magnetic cloud that has been eroded in a proportion similar to the erosion observed for the 18-20 October $1995 \mathrm{MC}$, i.e., equivalent to removing a $4 \mathrm{~h}$ long interval at the front. The duration of this compressed and eroded model MC is now $29 \mathrm{~h}$, consistent with that of the MC under study. Similarly, the magnetic field, speed, and $V B_{Z}$ are of the same order.

[19] Comparing the black and blue lines in Figure 6c, we observe that the mere compression of the MC leads to enhanced geo-effectiveness. The increase is $\sim 27 \%$ in the expected $D s t^{*}$ response. This is a known effect, which is of the same kind as that produced by compression at the rear of north-south MCs which are followed by high-speed streams, as highlighted by Fenrich and Luhmann [1998]. Of core interest here is the impact of erosion, which is observed by comparing the red curves with the blue and black curves. As can be seen, the erosion significantly lowers the $D s t^{*}$ response as compared to the similarly compressed but noneroded (blue lines) MC by $\sim 30 \%$. This brings the geomagnetic response to the level of the noneroded and noncompressed MC (black lines). This is only a coincidence given the setup of our calculations and would be different if using either a larger or a smaller compression, for instance.

[20] Despite the MC of 18-20 October 1995 looking fairly symmetric and its structure well preserved from a quick glance at the data (e.g., in Figure 3), detailed analysis reveals the probable removal of a significant amount of southward oriented magnetic field at its front owing to erosion, together with compressive effects. Had it been compressed but not eroded - even of such a small amount given the long duration of this $\mathrm{MC}$ - the geomagnetic response in $D s t^{*}$ would have been $\sim 30 \%$ larger, according to the model used here.

[21] The occurrence and amount of erosion is directly related to the orientation of the IMF in the solar wind prevailing ahead of the $\mathrm{MC}$ during propagation. If an $\mathrm{MC}$ similar to that observed on 18-20 October 1995 had impacted the Earth noneroded, it would have meant that the IMF ahead of it had an orientation predominantly similar to that in the front part of the $\mathrm{MC}$ during most of its propagation, that is, directed southward. A south-north MC impinging on the Earth noneroded is thus likely to be preceded by a sheath with compressed southward magnetic field, adding to the whole structure's geo-effectiveness. Furthermore, in the absence of erosion, the compression may be expected larger. In an essentially symmetric fashion, an eroded south-north polarity MC may have a lower impact not only because of shorter southward magnetic field duration in its front part but also because the sheath ahead is more likely to have a non-geoeffective northward orientation. These possibilities deserve dedicated studies.

\section{Estimates of the Radial Dependence of Magnetic Cloud Erosion}

[22] We restate here that the sole observation of ongoing magnetic reconnection at the front boundary of MCs demonstrates that erosion does occur, even if just in a small amount as observed locally. A key remaining issue is thus whether the reconnection rate can be sufficiently large during propagation to allow for the amount of magnetic flux erosion invoked in this and previous studies. Here we argue, based on simple calculations, that the reconnection rates and their profile in the inner heliosphere (up to $1 \mathrm{AU}$ ) are capable of producing the erosion on the order of those reported. This is thanks to the combination of relatively large Alfvén speeds in MCs and their leading sheaths (as compared to regular solar wind) and the increase in Alfvén speed upon approach to the Sun.

\subsection{Reconnection Rates at $1 \mathrm{AU}$}

[23] Local measures of the reconnection rate have been made for a few well-defined exhausts at 1 AU. Studies by Davis et al. [2006], Phan et al. [2006], and Wang et al. [2010] found reconnection rates of $0.02,0.03$, and $0.08 \mathrm{mV} / \mathrm{m}$ for magnetic field magnitudes/magnetic shears of $\sim 10 \mathrm{nT} / 145^{\circ}, \sim 11 \mathrm{nT} / 140^{\circ}$, and $\sim 14 \mathrm{nT} / 143^{\circ}$, respectively. While the two former cases occurred in rather regular slow solar wind, the latter was observed at the front of an MC. In addition to the magnetic field being a little higher, the latter case was also characterized by lower densities $\left(\sim 4-8 \mathrm{~cm}^{-3}\right.$ rather than $\left.\sim 8-14 \mathrm{~cm}^{-3}\right)$. These properties are thus consistent with the reconnection rate scaling with the Alfvén speed in the vicinity of the reconnection region [e.g., Cassak and 
Shay, 2007] or a hybrid version of it in the asymmetric cases [e.g., Borovsky and Hesse, 2007]. Note that the reconnection rate at the possible reconnection exhaust at the front of the 18-20 October $1995 \mathrm{MC}$ cannot be properly measured owing to the exhaust being less well defined than in the case studies reported above. We yet do not expect it to be particularly high at the time of in situ measurement since, despite higher magnetic fields, the magnetic shear is rather low $\left(\sim 34^{\circ}\right)$.

[24] As mentioned by McComas et al. [1988], internal MC magnetic field orientations are a priori unrelated to the upstream draped field orientations, so that approximately half of the time we would expect the two regions to have magnetic fields which are more antiparallel than parallel. In other words, and also in view of recent works suggesting that magnetic reconnection is ubiquitous even for low magnetic shear [Gosling and Szabo, 2008; Phan et al., 2010] (cf. also Swisdak et al. [2003, 2010] for dependence on plasma $\beta$ and shear), we may roughly assume that magnetic reconnection could be ongoing about half of the time during propagation. Note that magnetic fields in front of an MC that are parallel to the leading-edge MC magnetic field (and which may preclude magnetic reconnection about half of the time) will typically not hold in front of the MC but drape and diverge around it. This allows for new plasma with different magnetic field conditions to perpetually come into contact and reconnect with the front $\mathrm{MC}$ boundary during propagation, in a fashion akin to the Earth's magnetosphere (but cf. section 6 for further discussion).

[25] Given (1) reconnection rates of $0.02-0.08 \mathrm{mV} / \mathrm{m}$ for (2) magnetic fields of $\sim 10-14 \mathrm{nT}$ and (3) magnetic shears of $\sim 145^{\circ}$ in the aforementioned case studies, the reconnection rate for the higher Alfvén speeds expected at the front of most MCs (e.g., lower density and higher magnetic fields of $\sim 20 \mathrm{nT}$ as in the present 18-20 1995 event) may be assumed on the order of $0.1 \mathrm{mV} / \mathrm{m}$ locally at $1 \mathrm{AU}$ for half of the time when the magnetic shear is expected in the range of $90^{\circ}-180^{\circ}$ (i.e., more antiparallel than parallel magnetic fields). The average reconnection rate for random magnetic shear can thus be approximated to $\sim 0.05 \mathrm{mV} / \mathrm{m}$, making the strong assumption that no reconnection occurs for low magnetic shear (certainly low reconnection rates anyway). It should be noted that such an average reconnection rate is not exaggerated if one compares to the more commonly studied case of the Earth's magnetopause. With magnetic fields typically less than an order of magnitude higher ( $\sim 20 \mathrm{nT}$ at an MC front and $\sim 100 \mathrm{nT}$ at the magnetopause), reconnection rates at the Earth's magnetopause are easily in excess of $1.0 \mathrm{mV} / \mathrm{m}$ [Mozer and Retino, 2007], more than an order of magnitude higher than the cases reported in the solar wind for comparable magnetic shear ranges.

[26] Using the MC transit time to $1 \mathrm{AU}$ and the estimate of the eroded magnetic flux [cf. Ruffenach et al., 2012] for the present 18-20 October $1995 \mathrm{MC}$ event, we find an average reconnection rate in the range of $\sim 0.2-0.3 \mathrm{mV} / \mathrm{m}$. Whether using only the azimuthal flux or both the azimuthal and axial flux (which is lower), estimates of the average reconnection rates fall in the range of $0.12-0.51 \mathrm{mV} / \mathrm{m}$ when including the events from Ruffenach et al. [2012] and Dasso et al. [2006, 2007]. These estimates must be viewed as rough since they entail numerous hypotheses related to the MC crosssectional shape, length, (non-)expansion, etc. However, it is clear that the magnitude of the reconnection rates required during propagation to account for the erosion estimated in these studies remains on the order of 2-10 times larger than that expected to be able to occur in front of MCs at $1 \mathrm{AU}$, i.e., $\sim 0.05 \mathrm{mV} / \mathrm{m}$ on average according to the simple scaling argument given in the previous paragraph.

\subsection{Radial Profile of Alfvén Speeds and Average Reconnection Rates}

[27] However, Alfvén speeds and reconnection rates are expected to be higher closer to the Sun [e.g., Lavraud and Borovsky, 2008]. We thus provide here calculations based on simple analytical and empirical models of the radial profile of the Alfvén speed from the Sun to $1 \mathrm{AU}$, which are meant to reflect the expected profile of the reconnection rate during propagation.

[28] Most models of magnetic field and density - and thus Alfvén speed $\left(|B| / \sqrt{ } \rho \mu_{0}\right)$ - profiles as a function of heliospheric distance have the following simple analytical form: $\left\langle B>\right.$ or $\langle N\rangle=a R^{-b}$, where $R$ is the radial distance, $a$ is the magnetic field magnitude or density at $1 \mathrm{AU}$, and $b$ is the exponent representing the decay due to the three-dimensional expansion of the solar wind. We choose to use three models which cover a sufficient range of Alfvén speed profiles to provide an estimate of the errors associated with the use of such simple models.

[29] The first model was built by Leitner et al. [2007] from actual MC observations at various distances in the heliosphere (using mean values within the MC). In this model, the magnetic field is parameterized with $a=18.1 \mathrm{nT}$ and $b=1.64$, while the density is parameterized with $a=7.24$ $\mathrm{cm}^{-3}$ and $b=2.44$. Because our purpose is to compare the profiles, we use the same baseline values at $1 \mathrm{AU}$ for the parameter $a$ in the implementation of the following two models. The second model comes from regular non-CME solar wind observations and combines results from Mariani and Neubauer [1990] for the magnetic field and Schwenn [1990] for the density using Helios data. In this case, we have $b=1.56$ (the other value of 1.84 from Helios 2 is not used as it falls in the same ballpark of the other curves discussed later in Figure 7) for the magnetic field and $b=2.1$ for the density. Finally, the third model comes from purely theoretical considerations of solar wind expansion, so that $b=2.0$ for both the density and the radial magnetic field component and $b=1.0$ for the azimuthal magnetic field component [e.g., Osherovich et al., 1993; Farrugia et al., 1993]. Using these values, the magnetic field magnitude and Alfvén speed profiles are shown with the colored curves in Figures $7 \mathrm{a}$ and $7 \mathrm{~b}$, from the solar surface $(\sim 0.0046 \mathrm{AU})$ to $1 \mathrm{AU}$.

[30] Again, it is a known fact that the local Alfvén speed at mesoscales next to the reconnection region is a key ingredient to determining the reconnection rate [e.g., Cassak and Shay, 2007; Borovsky and Hesse, 2007], with, in the fast magnetic reconnection regime, the inflow speed $\sim 10 \%$ of the local Alfvén speed. The profiles in Figure $7 \mathrm{~b}$ thus provide a qualitative estimate of the reconnection rate profile during propagation in a statistical sense, assuming randomly variable local magnetic shear at the MC's front boundary and a constant $\mathrm{MC}$ velocity profile (which is reasonable between the Sun and the Earth, except very close to the Sun). Making these assumptions, the average reconnection rate experienced at the front of an $\mathrm{MC}$ during propagation scales as the average Alfvén speed during propagation. These average Alfvén speeds are given as the colored dashed lines 




Figure 7. Radial profiles of the (a) magnetic field strength, (b) Alfvén speed, and (c) cumulative distribution (in percentile) of the Alfvén speed up to $1 \mathrm{AU}$ in the heliosphere for three different models: black, blue, and red. The dashed lines in Figure $7 \mathrm{~b}$ show the average value of the Alfvén speed. The thin lines in Figure 7c highlight the values of the distribution at Mercury's orbit.

of constant value for each model in Figure 7b. They are between 1.6 (black case) and 3.5 (red case) times higher than the Alfvén speeds at $1 \mathrm{AU}$, for the given models. This is to be compared with the factor 2-10 difference between estimates of the average reconnection rates at the leading edge of $\mathrm{MCs}$ at $1 \mathrm{AU}(0.05 \mathrm{mV} / \mathrm{m})$ and reconnection rates required to account for the erosion observed in this and previous studies $(0.12-0.51 \mathrm{mV} / \mathrm{m})$ [Dasso et al., 2006, 2007; Ruffenach et al., 2012].

[31] The increase in Alfvén speed toward the Sun combined with the relatively large Alfvén speeds in MCs and their sheaths (compared to the more regular solar wind) are thus able to reconcile, to the first order and in a statistical sense, the rather low reconnection rates observed so far in situ at $1 \mathrm{AU}$ with the seemingly high rates required for the significant magnetic flux erosions reported.

[32] Therefore, in the event of highly favorable magnetic shear conditions during propagation-the potential occurrence of which remains to be studied-the process may lead to significantly larger erosions than those reported so far. In this context, it is worth citing the simulation work of Shiota et al. [2010]. Despite using an MHD approach which may not necessarily provide realistic reconnection rates, this work suggested the possibility of almost total MC erosion with highly favorable conditions. Although highly speculative, this opens the question as to whether this process could explain the fact that some coronal mass ejections do not possess an embedded, well-defined magnetic structure.

\subsection{Radial Profile of the Erosion Process}

[33] Finally, because reconnection rates are expected to increase on average on approach to the Sun, we may investigate where most of the erosion is susceptible to occur, again in a statistical sense assuming random magnetic shear during propagation and based on the given models. For that purpose, Figure $7 \mathrm{c}$ shows the accumulated Alfvén speed profiles, in percentile of the total accumulated Alfvén speed up to $1 \mathrm{AU}$ (assuming a constant velocity profile). Because the reconnection rate is assumed to scale with the Alfvén speed, and whatever the total amount of erosion discussed, these curves shed lights on where most of the erosion may occur. The black thin lines are meant to guide the eye and highlight that between 47 and $67 \%$ of the erosion is statistically expected to occur within Mercury's orbit, given the present models.

\section{Discussion of Some Limitations of the Approach}

[34] First, we ought to be reminded here that the magnetic flux erosion estimates using the direct method of Dasso et al. [2006], based on azimuthal magnetic flux asymmetry, relies on the use of methods (e.g., MVA as used here) which are known to have deficiencies. For instance, their reliability depends on the impact parameter (the minimal distance of the trajectory to the main axis, normalized to the MC radius) [e.g., Gulisano et al., 2007] and possible crossing of the MC legs [Owens et al., 2012], as well as on often present large-scale magnetic fluctuations within the MC. These facts render the analysis of the asymmetry of MCs quite uncertain. Multispacecraft data analyses such as in Ruffenach et al. [2012] permit to reduce these uncertainties. Future statistical studies of the erosion process will provide further information on this matter. For the 18 October 1995 studied here, however, the impact parameter is estimated low (0.07).

[35] Regarding MC properties, we have used a simple cylindrical Lundquist model for our calculations, while more complex shapes may exist [e.g., Mulligan and Russell, 2001; Owens, 2006; Savani et al., 2010]. The use of a simple model is sufficient for illustrating the impact of erosion to the first order as done here. What may affect the erosion process, however, is the impact of the MC shape on the plasma flows in the sheath [e.g., Siscoe and Odstrcil, 2008] and, in turn, on the ability to transport the reconnected magnetic flux to the side and away from the MC nose where reconnection is expected to continue. Such aspects are beyond the scope of the present study and deserve dedicated simulation work.

[36] The validity of the Dst model used here should be also mentioned. In addition to being valid for only storm strength with minimum $D s t>-150 \mathrm{nT}$, as determined by $O^{\prime}$ Brien and McPherron [2000], the model is semiempirical and has significant inherent statistical uncertainties. It remains that the erosion process should have an impact on the order of that presented here, on average, if applied to a sufficiently large set of events. In any case, there is no doubt that an eroded MC with shorter duration southward magnetic field at its front will be less geo-effective, independent of the Dst model which merely provides an estimate.

[37] The erosion process is directly dependent upon the magnetic shear at the front boundary during propagation and, therefore, on the orientation of the IMF in the solar wind prevailing ahead of the MC. Not only is this orientation very hard to predict even using state-of-the-art models that 
reconstruct the coronal magnetic field, but this orientation is strongly affected during propagation by compressional effects, large-scale Alfvén waves, the flux-tube texture of the solar wind, and turbulence at all scales. Despite being a major limiting factor, we do not foresee any simple way to estimate the distribution of magnetic shear at the MCs' front boundary during propagation.

[38] A significant assumption in the estimates of the erosion radial evolution comes from the fact that reconnection could be absent despite parameters such as the plasma $\beta$ and magnetic shear being favorable. This could owe, for instance, to the current sheet being too thick. However, MCs that expand into the ambient solar wind with higher speed should be able to drive the thinning of the front current sheet. In particular, in an analogy to magnetic reconnection at the Earth's magnetopause, one may speculate that compression and draping over $\mathrm{MC}$ boundaries ought to favor the occurrence of magnetic reconnection somewhere on its outer boundary at all times. Such considerations deserve future work.

[39] Finally, it should be noted that the modeled Alfvén speed values extrapolated close to the Sun (between 1000 and $10000 \mathrm{~km} / \mathrm{s}$ ) in Figure 7 are on the order of those given by dedicated models of the Alfvén speed profile in the near-Sun corona, in the $1000-5000 \mathrm{~km} / \mathrm{s}$ range [cf. Evans et al., 2008]. While the Leitner et al. [2007] extrapolation (black line in Figure 7b) is rather low for active regions in the low corona, the regular solar wind model (red line), on the other hand, seems to overestimate the magnetic field there. Overall, the use of these three different models, possibly both underestimating and overestimating actual profiles, provides a reasonably large set of uncertainties for the purpose of the present study.

\section{Conclusions}

[40] Albeit using simple models and calculations, the present study provides important new information regarding the geo-effectiveness and radial evolution of the process of $\mathrm{MC}$ erosion by magnetic reconnection. The main conclusions may be summarized as follows:

[41] 1. It is a fact that MC erosion occurs at the front boundary of $\mathrm{MCs}$, at least at times. The sole observation of reconnection exhausts at the front of some $\mathrm{MC}$ is a sufficient proof.

[42] 2. Estimates of the influence of such an erosion process for a given MC that occurred on 18 October 1995 show that the erosion may have led to a decrease in geo-effectiveness (as measured by the Dst index) on the order of $\sim 30 \%$.

[43] 3. A parametric study shows that this decrease may, in principle, be very large. However, whether the process is often significant will require future statistical study.

[44] 4. It is further highlighted that under favorable conditions (i.e., parallel sheath and MC magnetic field during most of the propagation), the combined effect of front compression and reduced (or lack thereof) magnetic erosion will constructively conspire to enhance the geo-effectiveness of MCs.

[45] 5. Conversely, extreme erosions may significantly affect the integrity of $\mathrm{MCs}$ and thus could explain the fact that some coronal mass ejections do not possess an embedded, well-defined magnetic structure.

[46] 6. Using simple models of the Alfvén speed radial profile in the solar wind and $\mathrm{MCs}$, the average reconnection rates expected during propagation should be between 1.6 and 3.5 larger than those measured at $1 \mathrm{AU}$, placing observed reconnection rates in the solar wind at $1 \mathrm{AU}$ in reasonable agreement with those estimated from the amount of erosion observed in this and previous case studies.

[47] 7. Finally, our calculations also suggest that a significant fraction of the erosion ought to occur within Mercury's orbit, but that yet up to $50 \%$ of the erosion may occur beyond it.

[48] Studies are underway to statistically examine the occurrence of this process over recent solar cycles. For instance, it is possible that the geo-effectiveness of the frontside erosion process may have solar cycle dependence, following the north-south/south-north MC polarity dependence [Mulligan et al., 1998]. Future inner heliospheric probes such as Solar Orbiter, Bepi-Colombo, and Solar Probe Plus will be ideal to further quantify this process.

[49] Acknowledgments. We thank the OMNI and WIND teams for providing the OMNI and WIND data to the community.

[50] Philippa Browning thanks the reviewers for their assistance in evaluating this paper.

\section{References}

Aguado, J., C. Cid, E. Saiz, and Y. Cerrato (2010), Hyperbolic decay of the Dst index during the recovery phase of intense geomagnetic storms, J. Geophys. Res., 115, A07220, doi:10.1029/2009JA014658.

Borovsky, J. E., and M. Hesse (2007), The reconnection of magnetic fields between plasmas with different densities: Scaling relations, Phys. Plasmas, 14, 102,309, doi:10.1063/1.2772619.

Burton, R. K., R. L. McPherron, and C. T. Russell (1975), An empirical relationship between interplanetary conditions and Dst, J. Geophys. Res., $80,4204-4214$.

Cassak, P. A., and M. A. Shay (2007), Scaling of asymmetric magnetic reconnection: General theory and collisional simulations, Phys. Plasmas, 14, 102,114, doi:10.1063/1.2795630.

Collier, M. R., et al. (2001), Reconnection remnants in the magnetic cloud of October 18-19, 1995: A shock, monochromatic wave, heat flux dropout, and energetic ion beam, J. Geophys. Res., 106, 15,985-16,000, doi:10.1029/2000JA000101.

Dasso, S., D. Gómez, and C. H. Mandrini (2002), Ring current decay rates of magnetic storms: A statistical study from 1957 to 1998, J. Geophys. Res., 107(A5), 1059, doi:10.1029/2000JA000430.

Dasso, S., C. H. Mandrini, P. Démoulin, and M. L. Luoni (2006), A new model-independent method to compute magnetic helicity in magnetic clouds, Astron. Astrophys., 455, 349-359.

Dasso, S., M. S. Nakwacki, P. Demoulin, and C. H. Mandrini (2007), Progressive transformation of a flux rope to an ICME, Sol. Phys., 244(1-2), 115-137, doi:10.1007/s11207-007-9034-2.

Davis, M. S., T. D. Phan, J. T. Gosling, and R. M. Skoug (2006), Detection of oppositely directed reconnection jets in a solar wind current sheet, Geophys. Res. Lett., 33, L19102, doi:10.1029/2006GL026735.

Démoulin, P., and S. Dasso (2009), Causes and consequences of magnetic cloud expansion, Astron. Astrophys., 498, 551-566.

Eriksson, S., et al. (2009), Asymmetric shear flow effects on magnetic field configuration within oppositely directed solar wind reconnection exhausts, J. Geophys. Res., 114, A07103, doi:10.1029/2008JA013990.

Evans, R. M., M. Opher, W. B. Manchester IV, and T. I. Gombosi (2008), Alfvén profile in the lower corona: Implications for shock formation, Astrophys. J., 687(2), 1355-1362.

Farrugia, C. J., L. F. Burlaga, V. Osherovich, I. G. Richardson, M. P. Freeman, R. P. Lepping, and A. Lazarus (1993), A study of an expanding interplanetary magnetic cloud and its interaction with the Earth's magnetosphere: The interplanetary aspect, J. Geophys. Res., 98, 7621-7632.

Farrugia, C. J., et al. (2001), A reconnection layer associated with a magnetic cloud, Adv. Space Res., 28(5), 759-764, doi:10.1016/S0273-1177(01)00529-4.

Fenrich, F. R., and J. G. Luhmann (1998), Geomagnetic response to magnetic clouds of different polarity, Geophys. Res. Lett., 25, 2999-3002.

Gosling, J. T. (1993), The solar flare myth, J. Geophys. Res., 98, 18,937-18,949, doi:10.1029/93JA01896.

Gosling, J. T., and A. Szabo (2008), Bifurcated current sheets produced by magnetic reconnection in the solar wind, J. Geophys. Res., 113, A10103, doi:10.1029/2008JA013473.

Gosling, J. T., S. J. Bame, D. J. McComas, and J. L. Phillips (1990), Coronal mass ejections and large geomagnetic storms, J. Geophys. Res., 17, 901-904. 


\section{LAVRAUD ET AL.: MAGNETIC CLOUD EROSION GEO-EFFECTIVENESS}

Gosling, J. T., R. M. Skoug, D. J. McComas, and C. W. Smith (2005), Direct evidence for magnetic reconnection in the solar wind near $1 \mathrm{AU}$, J. Geophys. Res., 110, A01107, doi:10.1029/2004JA010809.

Gosling, J. T., S. Eriksson, and R. Schwenn (2006), Petschek-type magnetic reconnection exhausts in the solar wind well inside $1 \mathrm{AU}$ : Helios, J. Geophys. Res., 111, A10102, doi:10.1029/2006JA011863.

Gulisano, A., S. Dasso, C. Mandrini, and P. Démoulin (2007), Estimation of the bias of the minimum variance technique in the determination of magnetic clouds global quantities and orientation, Adv. Space Res., 40, 1881-1890, doi:10.1016/j.asr.2007.09.001.

Hidalgo, M. A., C. Cid, A. F. Viñas, and J. Sequeiros (2002), A non-forcefree approach to the topology of magnetic clouds in the solar wind, J. Geophys. Res., 107(A1), 1002, doi:10.1029/2001JA900100.

Huttunen, K. E. J., S. D. Bale, and C. Salem (2008), Wind observations of low energy particles within a solar wind reconnection region, Ann. Geophys., 26, 2701-2710, doi:10.5194/angeo-26-2701-2008.

Janoo, L., et al. (1998), Field and flow perturbations in the October 18-19, 1995 , magnetic cloud, J. Geophys. Res., 103, 17,249-17,259, doi:10.1029/ 97JA03173.

Kawano, H., and T. Higuchi (1995), The bootstrap method in space physics: Error estimation for the minimum variance analysis, Geophys. Res. Lett. 22, 307-310, doi:10.1029/94GL02969.

Larson, D. E., et al. (1997), Tracing the topology of the October 18-20, 1995, magnetic cloud with $\sim 0.1-10^{2} \mathrm{keV}$ electrons, Geophys. Res. Lett., 24, 1911-1914.

Lavraud, B., and J. E. Borovsky (2008), Altered solar wind-magnetosphere interaction at low Mach numbers: Coronal mass ejections, J. Geophys. Res., 113, A00B08, doi:10.1029/2008JA013192.

Lavraud, B., et al. (2009), Observation of a complex solar wind reconnection exhaust from spacecraft separated by over $1800 R_{E}$, Sol. Phys., 256(1-2), 379-392, doi:10.1007/s11207-009-9341-x.

Leitner, M., C. J. Farrugia, C. Möstl, K. W. Ogilvie, A. B. Galvin, R. Schwenn, and H. K. Biernat (2007), Consequences of the force-free model of magnetic clouds for their heliospheric evolution, J. Geophys. Res., 112, A06113, doi:10.1029/2006JA011940.

Lepping, R. P., et al. (1997), The Wind magnetic cloud and events of October 18-20, 1995: Interplanetary properties and as triggers for geomagnetic activity, J. Geophys. Res., 102, 14,049-14,063, doi:10.1029/ 97JA00272.

Lundquist, S. (1950), Magneto-hydrostatic fields, Ark. Fys., 2(35), 361-365.

Mariani, F., and F. M. Neubauer (1990), The interplanetary magnetic field, in Physics of the Inner Heliosphere, vol. I, edited by R. Schwenn and E. Marsch, pp. 183, Springer, New York.

McComas, D. J., J. T. Gosling, D. Winterhalter, and E. J. Smith (1988), Interplanetary magnetic field draping about fast coronal mass ejecta in the outer heliosphere, J. Geophys. Res., 93, 2519-2526, doi:10.1029/ JA093iA04p02519.

Möstl, C., C. Miklenic, C. J. Farrugia, M. Temmer, A. Veronig, A. B. Galvin, and H. K. Biernat (2008), Two-spacecraft reconstruction of a magnetic cloud and comparison to its solar source, Ann. Geophys., 26, 3139-3152, doi:10.5194/angeo-26-3139-2008.

Mozer, F. S., and A. Retinò (2007), Quantitative estimates of magnetic field reconnection properties from electric and magnetic field measurements, J. Geophys. Res., 112, A10206, doi:10.1029/2007JA012406.

Möstl, C., M. Temmer, T. Rollett, C. J. Farrugia, Y. Liu, A. M. Veronig, M. Leitner, A. B. Galvin, and H. K. Biernat (2010), STEREO and Wind observations of a fast ICME flank triggering a prolonged geomagnetic storm on 5-7 April 2010, Geophys. Res. Lett., 37, L24103, doi:10.1029/2010GL045175.

Mulligan, T., and C. T. Russell (2001), Multispacecraft modeling of the flux rope structure of interplanetary coronal mass ejections: Cylindrical symmetric versus nonsymmetric topologies, J. Geophys. Res., 106 $10,581-10,596$.
Mulligan, T., C. T. Russell, and J. G. Luhmann (1998), Solar cycle evolution of the structure of magnetic clouds in the inner heliosphere, Geophys. Res. Lett., 25, 2959-2962.

O’Brien, T. P., and R. L. McPherron (2000), An empirical phase space analysis of ring current dynamics: Solar wind control of injection and decay, J. Geophys. Res., 105, 7707-7719.

Osherovich, V. A., C. J. Farrugia, and L. F. Burlaga (1993), Dynamics of aging magnetic clouds, Adv. Space Res., 13(6), 57-62.

Owens, M. J. (2006), Magnetic cloud distortion resulting from propagation through a structured solar wind: Models and observations, J. Geophys. Res., 111, A12109, doi:10.1029/2006JA011903.

Owens, M. J., P. Démoulin, N. P. Savani, B. Lavraud, and A. Ruffenach (2012), Implications of non-cylindrical flux ropes for magnetic cloud reconstruction techniques and the interpretation of double flux-rope events, Sol. Phys., 278(2), 435-446, doi:10.1007/s11207-012-9939-2.

Phan, T. D., et al. (2006), A magnetic reconnection X-line extending more than 390 Earth radii in the solar wind, Nature, 439(7073), 175-178, doi: 10.1038 /nature 04393

Phan, T. D., J. T. Gosling G. Paschmann, C. Pasma, J. F. Drake, M. Øieroset, D. Larson, R. P. Lin, and M. S. Davis (2010), The dependence of magnetic reconnection on plasma $\beta$ and magnetic shear: Evidence from solar wind observations, Astrophys. J. Lett., 719, L199-L203, doi:10.1088/ 2041-8205/719/2/L199.

Rouillard, P., B. Lavraud, N. R. Sheeley, J. A. Davies, L. F. Burlaga, N. P. Savani, C. Jacquey, and R. J. Forsyth (2010), White light and in situ comparison of a forming merged interaction region, Astrophys. J., $719(2)$, 1385-1392, doi:10.1088/0004-637X/719/2/1385.

Ruffenach, A., et al. (2012), Multispacecraft observation of magnetic cloud erosion by magnetic reconnection during propagation, J. Geophys. Res. 117, A09101, doi:10.1029/2012JA017624

Savani, N. P., M. J. Owens, A. P. Rouillard, R. J. Forsyth, and J. A. Davies (2010), Observational evidence of a coronal mass ejection distortion directly attributable to a structured solar wind, Astrophys. J. Lett., 714(1), L128-L132.

Schmidt, J. M., and P. J. Cargill (2003), Magnetic reconnection between a magnetic cloud and the solar wind magnetic field, J. Geophys. Res. 108(A1), 1023, doi:10.1029/2002JA009325.

Schwenn, R. (1990), Large-scale structure of the interplanetary medium, in Physics of the Inner Heliosphere, vol. I, edited by R. Schwenn and E. Marsch, pp. 99, Springer, New York.

Shiota, D., K. Kusano, T. Miyoshi, and K. Shibata (2010) Magnetohydrodynamic modeling for a formation process of coronal mass ejections: Interaction between an ejecting flux rope and an ambient field, Astrophys. J., 718, 1305-1314, doi:10.1088/0004-637X/718/2/1305.

Siscoe, G., and D. Odstrcil (2008), Ways in which ICME sheaths differ from magnetosheaths, J. Geophys. Res., 113, A00B07, doi:10.1029/ 2008JA013142.

Swisdak, M., B. N. Rogers, J. F. Drake, and M. A. Shay (2003), Diamagnetic suppression of component magnetic reconnection at the magnetopause, J. Geophys. Res., 108(A5), 1218, doi:10.1029/2002JA009726.

Swisdak, M., M. Opher, J. F. Drake, and F. Alouani Bibi (2010), The vector direction of the interstellar magnetic field outside the heliosphere, Astrophys. J., 710, doi:10.1088/0004-637X/710/2/1769.

Taubenschuss, U., N. V. Erkaev, H. K. Biernat, C. J. Farrugia, C. Möstl, and U. V. Amerstorfer (2010), The role of magnetic handedness in magnetic cloud propagation, Ann. Geophys., 28(5), 1075-1100, doi:10.5194/ angeo-28-1075-2010.

Wang, Y., F. Wei, X. Feng, S. Zhang, P. Zuo, and T. Sun (2010), Energetic electrons associated with magnetic reconnection in the magnetic cloud boundary layer, Phys. Rev. Lett., 105, 195,007, doi:10.1103/PhysRevLett.105.195007.

Zhang, G., and L. F. Burlaga (1988), Magnetic clouds, geomagnetic disturbances, and cosmic ray decreases, J. Geophys. Res., 93, 2511-2518. 